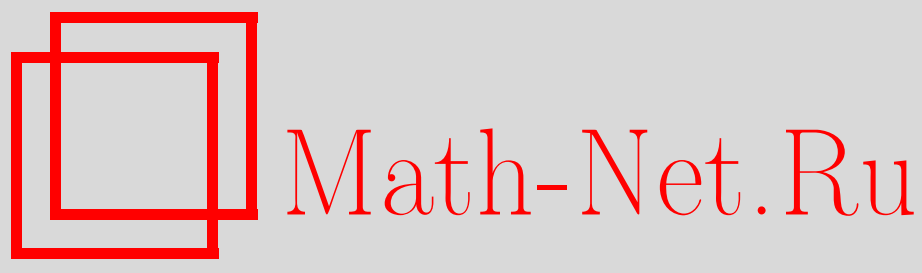

Обшероссийский математический портал

С. Я. Гриншпон, Вполне транзитивные однородно сепарабельные абелевы группы, Матем. заметки, 1997, том 62, выпуск 3, 471-474

DOI: https://doi.org/10.4213/mzm2771

Использование Общероссийского математического портала Math-Net.Ru подразумевает, что вы прочитали и согласны с пользовательским соглашением http://www.mathnet.ru/rus/agreement

Параметры загрузки:

IP: 3.85 .5 .30 
26 апреля 2023 г., 17:44:53

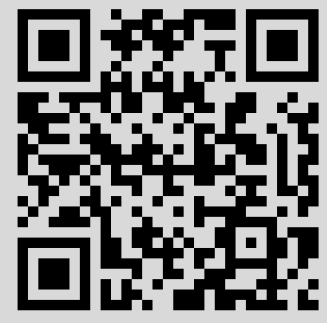




\section{ВПОЛНЕ ТРАНЗИТИВНЫЕ \\ ОДНОРОДНО СЕПАРАБЕЛЬНЫЕ АБЕЛЕВЫ ГРУППЫ}

\section{С. Я. Гриншпон}

Абелеву группу $G$ без кручения назовем однородно сепарабельной, если существует такое семейство $\mathbf{C}$ однородных прямых слагаемых этой групшы, что каждое конечное множество элементов группы $G$ можно вложить в прямое слагаемое этой группы, являющееся прямой суммой некоторых груп семейства С. Будем говорить в этом случае, что семейство $\mathbf{C}$ задает однородную сепарабельность групшы $G$.

Заметим, что однородно сепарабельными группами являются, в частности, вполне разложимые абелевы группы без кручения, сепарабельные абелевы групшы без кручения, однородно разложимые абелевы группы.

При изучении вполне характеристических подгрупп абелевых групш $G$ без кручения интерес представляют группы, в которьх каждая вполне характеристическая подгруппа имеет вид $G[v]=\{g \in G \mid \chi(g) \geqslant v\}$, где $v$-некоторая характеристика, т.е. последовательность, состоящая из целых неотрищательных чисел и символов $\infty$. Такие редуцированные групшы без кручения были названы в [1] $\chi$-группами. (Заметим, что при рассмотрении вполне характеристических подгрупп абелевых групп без кручения можно ограничиться редуцированными группами, так как ненулевая подгрупа $S$ абелевой групшы без кручения $A=R \oplus V$, где $R$ и $V$ - редуцированная и делимая части соответственно групшы $A$, вполне характеристична в $A$ тогда и только тогда, когда $S=R_{1} \oplus V$, где $R_{1}$ - вполне характеристическая подгрупша группы $R$ [1, лемма 3.3$\left.]\right)$. Всякая $\chi$-группа является вполне транзитивной группой, т.е. группой, в которой для любых двух элементов $x$ и $y$ таких, что $\chi(x) \leqslant \chi(y)(\chi(x), \chi(y)$ - характеристики элементов $x$ и $y$ соответственно) существует эндоморфизм $\varphi$ со свойством $\varphi(x)=y[1$, с. 63$]$ (в [1] такие группы назьвались транзитивными группами). Обратное в общем случае неверно. 
В настоящей статье получена характеризация вполне транзитивных однородно сепарабельных групп, в частности, установлено, что любая вполне транзитивная однородно сепарабельная групша является $\chi$-группой.

Пусть $a$ - некоторый элемент абелевой группы $G$ без кручения, $p$ - простое число. Обозначим через $h_{p}(a) \quad p$-высоту элемента $a$, а через $\chi(a), t(a)$ - характеристику и тип этого элемента соответственно. Далее пусть П - множество всех простых чисел, а $\pi(G)=\{p \in \Pi \mid p G \neq G\}$. Если $G$ - однородная группа, то $t(G)$ будет означать тип группы $G$, т.е. тип всех ее ненулевых элементов. Пусть $G$ - однородно разложимая абелева группа без кручения, т.е. $G=\bigoplus_{i \in I} A_{i}$, где $A_{i}$ - однородные групшы. Если собрать вместе компоненты $A_{i}$ одного и того же типа и взять их прямую сумму, то мы получим каноническое (наименьшее однородное [2, с. 212]) разложение $G=\bigoplus_{t \in T} G_{t}$, где $G_{t}-$ однородные групшы различных типов $t$.

ТЕорема. Пусть $G$ - однородно сепарабельная редуиированная группа, $\mathbf{C}=$ $\left\{A_{i}\right\}_{i \in I}$ - семейство однородных абелевых групп без кручения, задающих однородную сепарабельность группь $G, T$ - мнохсество типов групп $A_{i}(i \in I)$. Следующие условия әквивалентны:

1) $G$ - вполне транзитивная группа;

2) если $G_{1}$ - однородно разложимое прямое слагаемое группы $G u G_{1}=\bigoplus_{t \in T_{1}} G_{t}$ - его каноническое разложение, то все группы $G_{t}$ вполне транзитивны и $\pi\left(G_{t_{1}}\right) \cap \pi\left(G_{t_{2}}\right)=\varnothing$ npu $t_{1} \neq t_{2}\left(t_{1}, t_{2} \in T_{1}\right)$

3) кахсдая группа $A_{i}(i \in I)$ вполне транзитивна $и \pi\left(A_{i_{1}}\right) \cap \pi\left(A_{i_{2}}\right)=\varnothing$, если $t\left(A_{i_{1}}\right) \neq t\left(A_{i_{2}}\right)$

4) группа $G$ - однородно разложимия группа, причем $G_{t}=\{g \in G \mid t(g)=t\} \cup\{0\}$ - подгруппа группь $G$ для всякого $t \in T u G=\bigoplus_{t \in T} G_{t}$ - каноническое разложение группы $G$, в котором каждая группа $G_{t}$ вполне транзитивна, $и$ $\pi\left(G_{t_{1}}\right) \cap \pi\left(G_{t_{2}}\right)=\varnothing n p u t_{1} \neq t_{2}\left(t_{1}, t_{2} \in T\right)$

5) $G-\chi-$-2pynna.

ДокаЗАТЕЛьство. Эквивалентность 1) и 2) доказана в [1, следствие 2.15]. Покажем 1$) \Longrightarrow 3$ ). Каждая групп $A_{i}$ вполне транзитивна как прямое слагаемое вполне транзитивной групшы $[1$, следствие 2.4$]$. Пусть $t\left(A_{i_{1}}\right) \neq t\left(A_{i_{2}}\right)\left(i_{1}, i_{2} \in I\right)$. Положим для определенности $t\left(A_{i_{1}}\right) \ngtr t\left(A_{i_{2}}\right)$. Имеем $G=A_{i_{1}} \oplus B=A_{i_{2}} \oplus \mathbf{C}$. Пусть $0 \neq a \in A_{i_{2}}$, $a=a_{1}+b$, где $a_{1} \in A_{i_{1}}, b \in B$. Тогда если $a_{1} \neq 0$, то $\chi(a) \leqslant \chi\left(a_{1}\right)$, чего быть не может, так как $t\left(A_{i_{1}}\right) \ngtr t\left(A_{i_{2}}\right)$. Значит, $a_{1}=0$ и $A_{i_{2}} \subset B$. Имеем $B=A_{i_{2}} \oplus(\mathbf{C} \cap B)$ и поэтому $G=A_{i_{1}} \oplus A_{i_{2}} \oplus(\mathbf{C} \cap B) ; A_{i_{1}} \oplus A_{i_{2}}$ - однородно разложимая вполне транзитивная группа, следовательно, $\pi\left(A_{i_{1}}\right) \cap \pi\left(A_{i_{2}}\right)=\varnothing[1$, предложение 2.12$]$.

Установим 3$) \Longrightarrow 4)$. Покажем вначале, что $G_{t}=\{g \in G \mid t(g)=t\} \cup\{0\}$ - подгруппа группы $G(t \in T)$. Пусть $a_{1}, a_{2} \in G_{t}, a_{1} \neq 0, a_{2} \neq 0$. Так как $G$ - однородно сепарабельная группа и семейство групп $\left\{A_{i}\right\}_{i \in I}$ задает однородную сепарабельность этой группы, то $a_{1}$ и $a_{2}$ можно вложить в прямое слагаемое $A_{k_{1}} \oplus A_{k_{2}} \oplus \cdots \oplus A_{k_{s}}$ группы $G$ $\left(k_{j} \in I, j=\overline{1, s}\right)$, причем можно считать, что для каждой группы $A_{k_{j}}$ хотя бы один из элементов $a_{1}, a_{2}$ имеет в этой группе ненулевую координату. Хотя бы одно из слагаемых $A_{k_{j}}(j=\overline{1, s})$ имеет тип $t$, так как в противном случае из условия $\pi\left(A_{i_{1}}\right) \cap \pi\left(A_{i_{2}}\right)=\varnothing$ при $t\left(A_{i_{1}}\right) \neq t\left(A_{i_{2}}\right)\left(i_{1}, i_{2} \in I\right)$ имели бы $t\left(a_{1}\right) \neq t$ и $t\left(a_{2}\right) \neq t$. Пусть для определенности $t\left(A_{k_{1}}\right)=t$. Если для любого простого числа $p h_{p}\left(a_{1}\right)<\infty$, то в силу редуцированности группы $G$ имеем $t\left(A_{k_{j}}\right)=t\left(A_{k_{1}}\right), j=\overline{2, s}$. 
Пусть $\mathscr{P}\left(A_{k_{1}}\right)=\left\{p \mid p-\right.$ простое число и $\left.p A_{k_{1}}=A_{k_{1}}\right\}$. Рассмотрим случай, когда $\mathscr{P}\left(A_{k_{1}}\right) \neq 0$. Покажем, что и в этом случае $t\left(A_{k_{j}}\right)=t\left(A_{k_{1}}\right), j=\overline{2, s}$. Предположим противное: пусть существует индекс $k_{l}(l=\overline{2, s})$ такой, что $t\left(A_{k_{l}}\right) \neq t\left(A_{k_{1}}\right)$. Рассматривая элемент $a_{i}(i=1,2)$, имеюший ненулевую координату в групше $A_{k_{l}}$, получаем, что существует такое простое число $p \in \mathscr{P}\left(A_{k_{1}}\right)$, для которого $h_{p}\left(a_{i}\right)<\infty$, а это противоречит тому, что $t\left(a_{i}\right)=t\left(A_{k_{1}}\right)=t$.

Итак, $A_{k_{1}} \oplus A_{k_{2}} \oplus \cdots \oplus A_{k_{s}}$ - однородная группа типа $t$. Значит, поскольку $a_{1}-a_{2} \in$ $A_{k_{1}} \oplus A_{k_{2}} \oplus \cdots \oplus A_{k_{s}}$, то $t\left(a_{1}-a_{2}\right)=t$ и $a_{1}-a_{2} \in G_{t}$. Следовательно, $G_{t}=\{g \in G \mid$ $t(g)=t\} \cup\{0\}-$ подгрупша группы $G$ для любого $t \in T$. Понятно, что для всякого типа $t \in T G_{t} \cap \sum_{t^{\prime} \in T} G_{t^{\prime}}=0$. Значит, $\sum_{t \in T} G_{t}=\bigoplus_{t \in T} G_{t}$. Остается показать, что $G=\bigoplus_{t \in T} G_{t}$.

Пусть $g \in G$, тогда элемент $g$ вкладывается в прямое слагаемое групшы $G$, разложимое в прямую сумму некоторых групп из семейства $\mathbf{C}\left(\mathbf{C}=\left\{A_{i}\right\}_{i \in I}\right)$. Каждая группа семейства $\mathbf{C}$ содержится в некоторой групше $G_{t}$, и поэтому $g \in \bigoplus_{t \in T} G_{t}$.

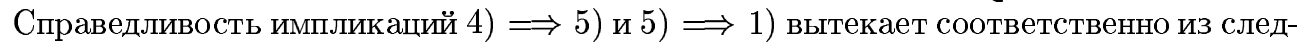
ствия 3.16 и предложения 2.1 работы [1].

Учитывая, что всякая абелева группа без кручения ранга 1 является вполне транзитивной групой, получаем такой результат.

СледСТВИЕ 1. Пусть $G$ - редуиированная сепарабельная абелева группа без кручения, $T$ - множество типов ее прямых слагаемых ранга 1. Следуюшие условия әквивалентны:

1) $G$ - вполне транзитивная группа;

2) если $G_{1}$ - вполне разложимое прямое слагаемое конечного ранга группь $G u$ $G_{1}=\bigoplus_{i=1}^{n} B_{i}$ - его разлохение в прямую сумму групп ранга 1 , то $\pi\left(B_{i_{1}}\right) \cap$ $\pi\left(B_{i_{2}}\right)=\varnothing \partial л я B_{i_{1}} ¥ B_{i_{2}}\left(i_{1}, i_{2}=\overline{1, n}\right)$;

3) для любых неизоморфных прямых слагаемых $A$ и $B$ ранга 1 группы $G$ имеем $\pi(A) \cap \pi(B)=\varnothing$

4) группа $G$ - однородно разлохсимая группа, причем $G_{t}=\{g \in G \mid t(g)=t\} \cup\{0\}$ - nодгруппа әруппы $G$ для всякого $t \in T u G=\bigoplus_{t \in T} G_{t}$ - каноническое разложение группь $G$, в котором каждая группа $G_{t}$ вполне транзитивна, $\pi\left(G_{t_{1}}\right) \cap \pi\left(G_{t_{2}}\right)=\varnothing$ npu $t_{1} \neq t_{2}\left(t_{1}, t_{2} \in T\right)$

5) $G-\chi$-гpynna.

Заметим, что эквивалентность условий 1) и 2) показана в [1, следствие 2.16].

Следуя [3], назовем абелеву групу $G$ без кручения группой тuпа $\mathscr{P}+$, если для некоторого простого числа $p$ она изоморфна $\mathbb{Z}_{p}$ - аддитивной групе всех целых $p$-адических чисел. Абелева група $G$ без кручения назьвается сепарабельной типа $\mathscr{P}+$, если любое ее конечное множество элементов содержится в некотором прямом слагаемом группы $G$, равном прямой сумме групп типа $\mathscr{P}+[3]$.

Сепарабельной группой типа $\mathscr{P}^{+}$является, в частности, всякая редуцированная абелева группа без кручения, на которой можно задать структуру унитарного $\mathbb{Q}_{p}^{*}$-модуля, где $\mathbb{Q}_{p}^{*}$ - кольцо целых $p$-адических чисел. Это вытекает из того, что всякий циклический подмодуль редуцированного $\mathbb{Q}_{p}^{*}$-модуля $G$ без кручения, порожденный элементом нулевой $p$-высоты, выделяется в $G$ прямым слагаемым [4, с. 294], [5, с. 52].

Учитывая, что всякая групп $\mathbb{Z}_{p}$ вполне транзитивна $[1$, с. 64], получаем 
СлЕДСТВИЕ 2. Пусть $G$ - сепарабельная группа типа Я尹P+. Тогда

1) $G$ - вполне транзитивная группа;

2) $G$ - однородно разложимая группа, имеющая каноническое разложение $G=\bigoplus_{p \in \Pi_{1}} G_{(p)}$, где $\Pi_{1} \subset \Pi$ и для всякого простого числа $p \in \Pi_{1}$ имеем $p G_{(p)} \neq G_{(p)}$ u $q G_{(p)}=G_{(p)}$ npu $q \in \Pi, q \neq p$

3) $G-\chi$-гpynna.

Абелева группа $A$ называется f.і.-корректной, если для всякой абелевой группы $B$ из того, что $A \cong B^{\prime}, B \cong A^{\prime}$, где $B^{\prime}, A^{\prime}$ - соответственно вполне характеристические подгрупшы групп $B$ и $A$, следует $A \cong B$ [6]. Так как всякая однородно разложимая вполне транзитивная абелева групша без кручения является f. i.-корректной [6, следствие 10], то получаем следуюший результат.

СлЕДСТВИЕ 3. Всякая редуцированная вполне транзитивная однородно сепарабельная группа (в частности, сепарабельная группа типа ЯР+ и вполне транзитивная сепарабельная группа без кручения) является f.і.-корректной әруппой.

\section{СПИСОК ЦИТИРОВАННОЙ ЛИТЕРАТУРЫ}

1. Гриншпон С.Я. О строении вполне характеристических подгрупп абелевых групп без кручения // Абелевы группы и модули. 1982. Т. 1. С. 56-92. 2. Фукс Л. Бесконечные абелевы группы. Т. 2. М.: Мир, 1977. 3. Прохазка Л . // Comment. Math. Univ. Carolin. 1967. № 1. P. 85-114. 4. Куликов Л. Я. // Тр. ММО. 1952. Т. 1. С. 247-326. 5. Kaplansky I. Infinite abelian groups. Michigan: Ann. Arbor, 1954. 6. Гриншпон С.Я. f. і.-корректность абелевых групп без кручения // Абелевы группы и модули. 1989. Т. 8. С. 65-79. 